\title{
Reconnection in Marginally Collisionless Accretion Disk Coronae
}

\author{
J. Goodman and D. Uzdensky \\ Princeton University Observatory, Princeton, NJ 08544
}

November 2, 2018

\begin{abstract}
We point out that a conventional construction placed upon observations of accreting black holes, in which their nonthermal X-ray spectra are produced by inverse comptonization in a coronal plasma, suggests that the plasma is marginally collisionless. Recent developments in plasma physics indicate that fast reconnection takes place only in collisionless plasmas. As has recently been suggested for the Sun's corona, such marginal states may result from a combination of energy balance and the requirements of fast magnetic reconnection.
\end{abstract}

Subject headings: accretion, accretion disks - magnetic fields - galaxies: activeX-rays: binaries

\section{Introduction}

The spectral states of X-ray binaries are distinguished by the relative strength of a soft quasi-thermal component and a harder power-law component. The soft component is attributed to an optically thick accretion disk, while the power law is usually ascribed to a hotter, geometrically thicker, and optically thinner distribution of plasma called the corona [see Zdziarski \& Gierliński (2004) for a recent review]. In some models the corona lies directly above the disk [the "sandwich model", Liang \& Price (1977); see also Uzdensky \& Goodman (2008) for more complete references], while in others the corona lies inside the inner edge of a radially truncated disk (e.g., Thorne \& Price 1975; Shapiro et al. 1976). During hard states at least, more energy is dissipated in the corona than in the disk. The coronal emission mechanism is usually identified as inverse comptonization of soft photons from the disk by hot, thermal coronal electrons (Eardley \& Lightman 1976), although synchrotron-selfCompton emission from relativistic electrons has also been proposed (e.g. Band \& Grindlay 1986; Field \& Rogers 1993). To explain the observed power-law spectra, the Compton parameter $y \equiv\left(4 k T_{e} / m_{e} c^{2}\right) \max \left(\tau, \tau^{2}\right)$ must be of order unity. Here $\tau \equiv n_{e} \sigma_{\mathrm{T}} H$ is the optical depth of the corona to electron scattering, $H$ its vertical scale height, and $T_{e}$ its electron 
temperature. On the other hand, the high-energy cutoff of the power law is typically above $100 \mathrm{keV}$, a significant fraction of the electron rest mass. It follows that the optical depth is also of order unity. A similar distinction between quasi-thermal and power-law components is observed in the continuum emission of AGN, except that the characteristic photon energy of the quasi-thermal component lies in the rest-rame UV or visible, as predicted by straightforward scalings with black-hole mass; the characteristic energy cut-off of the power-law component remains comparable to the electron rest mass.

It is generally belived that the solar corona is produced and maintained by magnetic reconnection events involving coronal loops. One of us has recently observed that the characteristic plasma density and magnetic field strength of the solar corona are such that reconnection layers should be marginally collisionless, in a sense made precise below, and that this may perhaps be a natural outcome of the requirement for fast reconnection [Uzdensky (2007); see also Cassak et al. (2006)]. Similar mechanism have also been shown to work in coronae of other stars (Cassak et al. 2008). We will show that a modest theoretical extrapolation beyond the framework summarized in the previous paragraph leads to the conclusion that disk coronae are also marginally collisionless, and that this may explain why disk coronae are able both to build up large stores of magnetic free energy and to dissipate them efficiently.

\section{Collisional and collisionless reconnection}

In this section, for the sake of completeness, we rehearse some points already made more fully by Uzdensky (2007). The upshot is the conjecture that under astrophysical conditions, fast Petschek-like reconnection occurs if and only if the Sweet-Parker thickness (1) is smaller than the ion skin depth (2).

In Sweet-Parker reconnection (Sweet 1958; Parker 1957), reconnecting field lines are brought together at a speed $V$ related to the Alfvén speed $V_{\mathrm{A}} \equiv B / \sqrt{4 \pi \rho}$ by $V \approx V_{\mathrm{A}} S^{-1 / 2}$, where $S \equiv L V_{\mathrm{A}} / \eta$ is the (dimensionless) Lundquist number based on the larger dimension of the reconnection layer, $L$, and the plasma magnetic diffusivity, $\eta$. It is presumed that $L$ is a macroscopic length characteristic of the size of the system: for example, the radius of curvature of a reconnecting magnetic loop. In most astrophysical situations, $S \gg 1$, meaning that the plasma is an excellent conductor on macroscopic scales, whence $V / V_{\mathrm{A}}$ is very small. Also, the theory predicts that thickness of the layer is

$$
\delta_{\mathrm{SP}}=L S^{-1 / 2}=\sqrt{\frac{\eta L}{V_{\mathrm{A}}}} .
$$


Sweet-Parker reconnection, though fairly well understood, is far too slow to explain solar flares, where reconnection is clearly fast in the sense that $V / V_{\mathrm{A}} \gtrsim 10^{-2} \gg S^{-1 / 2}$. The classical Petschek model was invented to solve this problem (Petschek 1964). In Petschek reconnection, the width $\delta$ of the reconnection region is not very much smaller than its length, $\delta \lesssim 0.1 L$; this yields fast reconnection because mass conservation and force balance imply $V / V_{\mathrm{A}} \approx \delta / L$. Recent numerical simulations, analytical arguments, and laboratory evidence indicate that in conventional one-fluid resistive magnetohydrodynamics (MHD), however, reconnection follows the Sweet-Parker rather than the Petschek model [see Uzdensky (2007) and references therein]. By "conventional" we mean that the mean free path of the current carriers is small compared to all macroscopic scales of interest, that $\eta$ is either constant or governed by two-body collisions among the carriers, and that two-fluid effects, such as the Hall effect, are unimportant. It has been suggested that something like Sweet-Parker reconnection might proceed much more quickly in a highly turbulent, fully three-dimensional

MHD because the reconnection layer could then be fractal (Lazarian \& Vishniac 1999), but as yet there is no clear evidence for this from laboratory plasmas or simulations.

Something like Petschek's fast reconnection is however observed when the reconnection layer is collisionless, so that resistive MHD is not strictly valid within the layer. The relevant condition is typically $\delta_{\mathrm{SP}}<d_{i}$, where $d_{i}$ is the ion collisionless skin depth, defined by

$$
d_{i} \equiv \frac{c}{\omega_{p i}}=\left(\frac{m_{i} c^{2}}{4 \pi Z_{i}^{2} e^{2} n_{i}}\right)^{1 / 2}
$$

and $\omega_{p i}$ is the ion plasma frequency (e.g., Cassak et al. 2006; Yamada et al. 2006; Uzdensky 2007). Hereafter we will assume that most of the ions are protons, so that $m_{i}=m_{p}$ and $Z_{i}=1$. The ion skin depth characterizes the scale below which inertial effects dominate the response of the ions to an electric field. Since $\delta_{\mathrm{SP}}$ involves $\eta$, which is normally governed by collisions (but see the discussion of inverse-Compton drag below), the condition $\delta_{\mathrm{SP}}<d_{i}$ can be restated in terms of the electron mean free path, as $\lambda_{e, \text { mfp }}>L\left(\beta_{e} m_{e} / m_{i}\right)^{1 / 2}$, where $\beta_{e}$ is the ratio of the electron pressure within the reconnection layer to the magnetic pressure outside it (Yamada et al. 2006). This condition highlights the role of plasma collisionality in determining the regime of the reconnection process (Uzdensky 2007).

\section{Collisionality of disk coronae}

In this paper we adopt the popular view that, just as the solar corona, accretion disk coronae (ADCe) are heated by numerous reconnection events (flares) involving coronal magnetic loops (e.g., Galeev et al. 1979; Field \& Rogers 1993; di Matteo 1998; Merloni \& Fabian 2002; Uzdensky \& Goodman 2008). Following the conjecture posed by $\S$ 2, our agenda now is 
to estimate the ratio $\delta_{\mathrm{SP}} / d_{i}$ in accretion disk coronae using the phenomenology summarized in $\S 1$.

It is useful to characterize the accreting system by dimensionless parameters. We have already introduced the optical depth, $\tau$, of the corona. The electron temperature will be represented by $\vartheta_{e} \equiv k T_{e} / m_{e} c^{2}$, and the ratio of the coronal ion pressure to magnetic stress by $\beta_{i}$. The dimensionless accretion rate is $\dot{m} \equiv \dot{M} c^{2} / L_{\mathrm{E}}$, scaled by the Eddington luminosity $L_{\mathrm{E}}=4 \pi G M m_{i} c / \sigma_{\mathrm{T}}$. A dimensionless radial coordinate $r \equiv R / R_{\mathrm{S}}$ scaled by the Schwarzschild radius $R_{\mathrm{S}}=2 G M / c^{2}$. The aspect ratio of the corona is $h \equiv H / R$. We do not distinguish between the scale heights of the magnetic stress and of the coronal plasma. The scale height of the cooler disk plasma, which is $\sim \dot{m} R_{S}$ if the disk is radiationpressure dominated, is presumed to be $\ll H$. Equilibrium along coronal field lines requires $k\left(T_{e}+T_{i}\right) \approx G M m_{i} H^{2} / R^{3}$; this becomes $\left(T_{i} / T_{e}\right)+1 \approx\left(m_{i} / m_{e}\right) h^{2} r / \vartheta_{e}$ in our dimensionless variables. Since $h, r$, and $\vartheta_{e}$ are all thought to be $O(1)$ where the corona is most luminous, a strongly two-temperature plasma is indicated: $T_{i} \gg T_{e}$.

To estimate the coronal magnetic field, we assume that the "viscous" torque that balances inward advection of angular momentum in steady state is magnetic, and that a fraction $f \leq 1$ of this torque is transmitted through the corona. In the sandwich model, the torque is ultimately exerted on the disk since the footpoints of the coronal field and most of the rotational inertia are rooted there. It seems likely that $f$ is closer to unity than to zero when the nonthermal power law dominates the spectrum Merloni \& Fabian (2002). Then $f \dot{M} \sqrt{G M R} \approx 4 \pi R^{2} H\left\langle-B_{R} B_{\phi} / 4 \pi\right\rangle_{\text {corona. }}$. If the opacity due to pairs is not dominant, so that $n_{e} \approx n_{p}$, this leads to

$$
\frac{V_{\mathrm{A}}^{2}}{c^{2}} \gtrsim f \dot{m} \tau^{-1} r^{-3 / 2}
$$

in dimensionless variables. We have written this as an inequality in case the magnetic pitch angle $\tan ^{-1}\left(B_{R} / B_{\phi}\right) \ll 1$, but we expect it to be an approximate equality. Next, hydrostatic balance along the field lines yields $V_{S}^{2} \approx h^{2} c^{2} / r$ for the coronal sound speed based on the gas pressure, so

$$
\beta \equiv \frac{V_{S}^{2}}{V_{\mathrm{A}}^{2}} \lesssim \tau h^{2}(f \dot{m})^{-1} r^{1 / 2}
$$

Here $\beta \equiv \beta_{i}+\beta_{e} \approx \beta_{i}$.

We take the magnetic diffusivity at the Spitzer value based on the electron temperature, presuming that $T_{p} \lesssim\left(m_{p} / m_{e}\right) T_{e}$ so that electrons rather than ions carry most of the current:

$$
\eta_{\mathrm{S}} \approx \vartheta_{e}^{-3 / 2} c r_{e} \ln \Lambda
$$

where $r_{e} \equiv e^{2} / m_{e} c^{2}$ is the classical radius of the electron. The relevant electron temperature entering (15) is the temperature within the Sweet-Parker reconnection layer just before the 
onset of fast reconnection. Importantly, because of the intense dissipation in the layer, this temperature may be as high as that obtained during the subsequent fast reconnection phase: while the speed at which the field lines approach one another is very different for the two modes of reconnection, the magnetic energy dissipated per unit mass of plasma entering the region is approximately the same. Furthermore, because of the rapid Compton cooling of the coronal electrons following a fast reconnection event, this reconnection-layer temperature can be much higher than the ambient coronal electron temperature. Thus, the covering fraction of the hot $\left(T_{e} \sim 100 \mathrm{keV}\right)$ corona may be very small, consistent with observations (Haardt et al. 1994; Stern et al. 1995; Nandra \& Papadakis 2001).

Alternatively, at high luminosities the mobility of the electrons can be dominated by Compton drag rather than Coulomb collisions. If Coulomb collisions with the ions could be entirely neglected, then the drift velocity of the (non-relativistic) electrons with respect the rest frame of the radiation field would become, in steady state, $\boldsymbol{v}_{e}=-(3 / 4)\left(\mathrm{ce} / \sigma_{\mathrm{T}} U_{\mathrm{rad}}\right) \boldsymbol{E}$, where $U_{\text {rad }}$ is the energy density in the radiation field and $\boldsymbol{E}$ is the electric field in that frame. The contribution of the electrons to the conductivity would then become $\sigma_{\mathrm{C}}=$ $(3 / 4) c e^{2} n_{e} / \sigma_{\mathrm{T}} U_{\text {rad }}$ (if the electrons are magnetized, then the conductivity becomes a tensor, but this formula for $\sigma_{\mathrm{C}}$ still relates the component of the electron current parallel to the magnetic field to the parallel component of $\boldsymbol{E})$. Expressed in our dimensionless variables, the corresponding resistivity $\eta_{\mathrm{C}}=c^{2} / 4 \pi \sigma_{\mathrm{C}}$ would then be

$$
\eta_{\mathrm{C}} \approx \frac{c \sigma_{\mathrm{T}} U_{\mathrm{rad}}}{3 \pi e^{2} n_{e}} \sim \frac{m_{p}}{m_{e}} \frac{\epsilon \dot{m} h}{r} c r_{e},
$$

where $\epsilon \equiv L / \dot{M} c^{2}$ is the radiative efficiency of accretion, and we have taken the photon energy density to be $U_{\text {rad }} \sim \tau L / 4 \pi R^{2} c \sim\left(\epsilon \dot{m} \tau / r^{2}\right)\left(m_{p} c^{2} / R_{S} \sigma_{T}\right)$ with $\tau \gtrsim 1$. A relativistic generalization of the first form of eq. (6) has been given by van Oss et al. (1993).

Since each positron contributes in the same way as an electron to the conductivity and to the optical depth, the estimate (6) for Compton diffusivity is unaffected by the presence of pairs, at least to the extent that their density in the corona is approximately uniformbut note that it may be enhanced in reconnection regions. We should consider whether the ions may dominate the conductivity, despite their greater inertia, when the leptons are immobilized by Compton drag. For an infinite and homogeneous electron-ion plasma, in fact, the DC (i.e., static) resistivity would be given by the Spitzer formula (5) regardless of the radiation field. This can be seen by considering the forces on the electrons and ions. Electric fields exert equal and opposite forces on the two charged species, whereas to a good approximation, radiation drag acts only on the electrons. Therefore in steady state, charge 
neutrality and force balance require the net Compton drag on the electrons to vanish 1 . Hence the mean velocity of the electrons should vanish in the rest frame of the radiation field. If momentum exchange between the electrons and ions is due solely to Coulomb encounters, then for a given electric field, the same drift velocity between the two species results as if the radiation field were absent, and hence the same conductivity. However, the ions probably cannot reach steady state during reconnection in an actual disk corona. While the electron mean free path corresponding to (5) is substantially smaller than the coronal scale height, $\lambda_{\text {m.f.p. }} / H \approx \vartheta_{e}^{2} /(\tau \ln \Lambda) \lesssim 10^{-2}$ for standard parameters, the collisional mean free path of the protons is larger by the factor $m_{p} / m_{e}$ and hence is $>H$. Thus the ions are not expected to reach their full drift velocity before leaving the reconnection layer, whose length is presumably $<H$; the ions decelerate only when they collide with the disk. So the contribution of the ions to the current is probably less than that of the electrons.

In short, we expect that the effective diffusivity for collisional reconnection is approximately the larger of $\eta_{\mathrm{S}}$ and $\eta_{\mathrm{C}}$. Comparing equations (5) and (6), we see that the ratio of Compton to Spitzer resistivities is roughly

$$
\frac{\eta_{C}}{\eta_{S}} \sim \frac{\theta_{e}^{3 / 2}}{\ln \Lambda} \frac{U_{\mathrm{rad}}}{n m_{e} c^{2}} \sim \frac{1}{\ln \Lambda} \frac{m_{p}}{m_{e}} \dot{m} \epsilon h \theta_{e}^{3 / 2} r^{-1} .
$$

With $\ln \Lambda \sim 20, \vartheta_{e} \sim 0.2$, and $\epsilon \sim 0.1$, the contributions (5) and (6) to the total resistivity may often be comparable near the inner edge of the disk, but the Spitzer resistivity probably dominates at larger radii because $\eta_{\mathrm{S}} / \eta_{\mathrm{C}} \propto r \vartheta_{e}^{-3 / 2}$.

If one takes the scale height $H=h r R_{\mathrm{S}}$ as the characteristic macroscopic length, the coronal Lundquist number based on the Spitzer resistivity becomes

$$
S_{\mathrm{S}}=\frac{H V_{\mathrm{A}}}{\eta_{S}} \sim\left(\frac{R_{\mathrm{S}}}{r_{e} \ln \Lambda}\right) f^{1 / 2} \dot{m}^{1 / 2} \tau^{-1 / 2} \vartheta_{e}^{3 / 2} h r^{1 / 4} .
$$

Similarly, the Lundquist number based on the Compton resistivity is

$$
S_{\mathrm{C}}=\frac{H V_{\mathrm{A}}}{\eta_{C}} \sim\left(\frac{R_{\mathrm{S}}}{r_{e}}\right) \frac{m_{e}}{m_{p}} f^{1 / 2} \dot{m}^{-1 / 2} \tau^{-1 / 2} \epsilon^{-1} r^{5 / 4} .
$$

All of the dimensionless factors except the first are expected to be of order unity, but $R_{\mathrm{S}} /\left(r_{e} \ln \Lambda\right) \sim 10^{17}\left(M / M_{\odot}\right)$ with $\ln \Lambda \approx 20$. The coronae of accreting black holes are

\footnotetext{
${ }^{1}$ We are ignoring gravity, which acts mainly on the ions. The electric field required to keep the electrons and ions from separating gravitationally is a potential field, whereas the electric fields associated with time derivatives of magnetic flux have nonzero curl. Potential fields do not affect the present discussion.
} 
therefore very, very deeply within the ideal-MHD regime, and Sweet-Parker reconnection is completely negligible. The ion skin depth $d_{i}=\left(m_{p} c^{2} / 4 \pi e^{2} n_{i}\right)^{1 / 2}$ becomes

$$
d_{i} \approx\left(\frac{m_{p}}{m_{e}} \frac{h r}{\tau}\right)^{1 / 2} \sqrt{r_{e} R_{\mathrm{S}}}
$$

if we take $n_{i} \approx n_{e}$ as before. This shows that the characteristic scale for fast reconnectionthe thickness of the current sheet - is approximately the geometric mean between two fundamental physical scales relevant to electromagnetic processes near a black hole: the size of the black hole and the classical electron radius.

Thus, we have the following expression for the collisionality parameter,

$$
\begin{aligned}
& \frac{\delta_{\mathrm{SP}}}{d_{i}} \sim \sqrt{\frac{m_{e}}{m_{p}} \ln \Lambda} f^{-1 / 4} \dot{m}^{-1 / 4} \tau^{3 / 4} \vartheta_{e}^{-3 / 4} r^{3 / 8}, \quad \text { if } \eta=\eta_{S}, \\
& \frac{\delta_{\mathrm{SP}}}{d_{i}} \sim \epsilon^{1 / 2} f^{-1 / 4} \dot{m}^{1 / 4} \tau^{3 / 4} h^{1 / 2} r^{-1 / 8}, \quad \text { if } \eta=\eta_{C} .
\end{aligned}
$$

Recall that reconnection is conjectured to be very slow when this ratio is $>1$ (the collisional, conventional MHD regime) but can be fast (reconnecting field lines approaching at speeds $\sim V_{\mathrm{A}}$ rather than $\sim V_{\mathrm{A}} S^{-1 / 2}$ ) when the ratio is less than unity.

\section{Discussion}

Marginal collisionality may explain why the particular combination of dimensionless parameters that enters eq. (11) should be of order unity, but it does not explain why the individual factors are also of order unity: in particular, the coronal optical depth $(\tau)$ and dimensionless temperature $\left(\vartheta_{e}\right)$. Additional constraints on these parameters have long been recognized. The combinations $\epsilon \dot{m}, f$, and $r^{-1}$ should be $<1$ for physical reasons, and it is not surprising that sources selected for high luminosity and prominent nonthermal emission approach these limits. It is plausible that relativistic corrections to Compton scattering and pair production explain why $\vartheta_{e} \lesssim O(1)$. In conjunction with these more familiar constraints, the condition $\delta_{\mathrm{SP}} \sim d_{i}$ then "explains" why $\tau \sim O(1)$. On the other hand, one might argue that there are other reasons why $\tau \sim O(1)$ : for example, the Thompson optical depth is about unity near $r=1$ in spherical accretion at the free-fall speed with $\dot{m}=1$ (e.g., Rees 1984). On this view, our result $\delta_{\mathrm{SP}} \sim d_{i}$ is merely a numerical coincidence. At the very least, however, the considerations of this paper indicate that reconnection in ADC is likely to occur within the marginally collisionless regime.

Taken together with equations (3) and (10), $\tau \sim O(1)$ implies that inner parts of ADCe of accreting black holes accreting near Eddington limit are characterized by the following 
fiducial values for plasma density, magnetic field strength, and the fast reconnection scale, which only depend on the black hole mass:

$$
\begin{aligned}
n_{*} & =\left(\sigma_{T} R_{g}\right)^{-1} \simeq 10^{19} \mathrm{~cm}^{-3} \mathrm{~m}^{-1}, \\
B_{*} & =c \sqrt{4 \pi n_{*} m_{p}} \simeq 4 \cdot 10^{8} \mathrm{~m}^{-1 / 2} \mathrm{G}, \\
d_{i *} & =\left(4 \pi n_{*} r_{i}\right)^{-1 / 2} \simeq 10^{-2} \mathrm{~m}^{1 / 2} \mathrm{~cm},
\end{aligned}
$$

where $m \equiv M / M_{\odot}$.

Most of the observational evidence for ADC pertains to the innermost part of the disk, where the physical parameters are comparable to those in equations (12)-(14). However, the outer parts may also have coronae. The hypothesis of marginal collisionality would then constrain the radial dependence of coronal properties. Equation (11) leads one to expect that $\tau$ or $\tau / \vartheta_{e}$ should vary as $r^{-1 / 2}$ in a given source. (In the second alternative on the right-hand side of that equation, $\tau^{1 / 4}$ replaces $\tau^{3 / 4}$ where $\tau<1$ because the radiation energy density becomes independent of $\tau$.) It would be interesting to explore the consequences of this for eclipsing systems such as the white dwarf binary OY Car (Wood et al. 1989), for example, where it may be possible to constrain the radial extent and structure of the X-ray-emitting region (Naylor et al. 1988; Ramsay et al. 2001); we expect that disk coronae should not be limited to black-hole systems.

The train of thought in this paper calls attention to some important issues. For example, how does magnetic reconnection proceed with marginally relativistic electrons and much hotter, perhaps also marginally relativistic, ions? How is it affected by the presence of a strong radiation background? What is the effect of pair creation on the reconnection process? What fraction of the magnetic energy released in the reconnection process and associated shocks goes to thermal electrons and nonthermal electrons? Does an appreciable fraction of the dissipated energy go into the ions, as appears to be the case for flares in the solar corona (e.g., Ramaty et al. 1995; Lin et al. 2003; Benz 2008)? What is the fate of the energy that goes to the ions? Whereas the main cooling mechanism for coronal electrons is probably Comptonization, the ions must cool (if at all) by other means. Some of their energy may be advected to infinity or into the event horizon in winds or plasmoids (i.e., disconnected loops of field and plasma). But the hot ions on closed field lines that are still tied to the slowly accreting disk seem likely to cool by collisions with the disk itself, unless this is inhibited by magnetic mirroring. Thus, ion thermal conduction, in addition to the direct irradiation by hard X-ray coronal emission, can be an imporant channel for transporting the energy dissipated in the corona down to the cool accretion disk. An important question then is, what are the main stopping mechanisms for hot coronal ions entering the much colder disk plasma and what are their observable signatures? 
We leave these interesting questions for future study, but it seems necessary to address a more central issue here: What are the feedback mechanisms that maintain the corona in a state of marginal collisionality? For the solar corona, Uzdensky (2007) proposed that the answer lies in the effect of reconnection on the balance between coronal heating and cooling. If the plasma density in the corona becomes large enough so that $\delta_{\mathrm{SP}}>d_{i}$ (i.e., the collisional regime), then reconnection becomes extremely slow and the rate of heating by magnetic energy dissipation falls sharply; the plasma then cools and drains down the field lines onto the photosphere until marginal collisionality is restored. Conversely, as the plasma density drops, fast reconnection converts magnetic energy into X-rays and energetic particles that evaporate photospheric gas into the corona. Should the coronal density nevertheless fall to an extremely low value and the coronal magnetic field become potential (i.e., current-free: $\boldsymbol{\nabla} \times \boldsymbol{B}=0$ ) through efficient reconnection, the field will offer less resistance to plasma-laden flux loops emerging from the turbulent photosphere. We expect that feedback mechanisms similar to these should operate in accretion disk coronae, but there may be some new effects due to the high luminosity and relativistic conditions. For example, pair creation inside the reconnection region may substantially increase the plasma density, thereby making the layer more collisional and inhibiting fast reconnection.

Finally, it is interesting to note that the coronal scale-height cancels when the Spitzer resistivity dominates. In fact, the first alternative in equation (11) would apply to the disk itself if the parameters $\left(f, \tau, \vartheta_{e}\right)$ were replaced by the torque fraction, optical depth, and dimensionless electron temperature of the disk. We expect $\tau_{\text {disk }} \gtrsim 1$ and $\vartheta_{e \text {,disk }} \ll 1$, so the disk is probably collisional and therefore, we conjecture, incapable of fast reconnection. The conclusion is stronger for more massive accretors (i.e., AGN rather than X-ray binaries) because the characteristic disk temperature at a fixed Eddington fraction $\dot{m}$ varies as $M^{-1 / 4}$, whereas the optical depth of the disk is independent of $M$ for a given $\dot{m}$ and Shakura-Sunyaev

$\alpha$ parameter. Yet even AGN show powerlaw X-ray spectra that suggest $\vartheta_{e} \gtrsim 0.1$, so they probably have active coronae.

We would like to acknowledge fruitful discussions with A. Socrates.

This work is supported by National Science Foundation Grant No. PHY-0215581 (PFC: Center for Magnetic Self-Organization in Laboratory and Astrophysical Plasmas).

\section{REFERENCES}

Band, D. L. \& Grindlay, J. E. 1986, ApJ, 308, 576 
Benz, A. O. 2008, Living Reviews in Solar Physics, 5, 1

Cassak, P. A., Drake, J. F., \& Shay, M. A. 2006, ApJ, 644, L145

Cassak, P. A., Mullan, D. J., \& Shay, M. A. 2008, ApJ, 676, L69

di Matteo, T. 1998, MNRAS, 299, L15+

Eardley, D. M. \& Lightman, A. P. 1976, Nature, 262, 196

Field, G. B. \& Rogers, R. D. 1993, ApJ, 403, 94

Galeev, A. A., Rosner, R., \& Vaiana, G. S. 1979, ApJ, 229, 318

Haardt, F., Maraschi, L., \& Ghisellini, G. 1994, ApJ, 432, L95

Lazarian, A. \& Vishniac, E. T. 1999, ApJ, 517, 700

Liang, E. P. T. \& Price, R. H. 1977, ApJ, 218, 247

Lin, R. P., Krucker, S., Hurford, G. J., Smith, D. M., Hudson, H. S., Holman, G. D., Schwartz, R. A., Dennis, B. R., Share, G. H., Murphy, R. J., Emslie, A. G., JohnsKrull, C., \& Vilmer, N. 2003, ApJ, 595, L69

Merloni, A. \& Fabian, A. C. 2002, MNRAS, 332, 165

Nandra, K. \& Papadakis, I. E. 2001, ApJ, 554, 710

Naylor, T., Bath, G. T., Charles, P. A., Hassal, B. J. M., Sonneborn, G., van der Woerd, H., \& van Paradijs, J. 1988, MNRAS, 231, 237

Parker, E. N. 1957, J. Geophys. Res., 62, 509

Petschek, H. E. 1964, in The Physics of Solar Flares, ed. W. N. Hess, 425-+

Ramaty, R., Mandzhavidze, N., Kozlovsky, B., \& Murphy, R. J. 1995, ApJ, 455, L193+

Ramsay, G., Poole, T., Mason, K., Córdova, F., Priedhorsky, W., Breeveld, A., Much, R., Osborne, J., Pandel, D., Potter, S., West, J., \& Wheatley, P. 2001, A\&A, 365, L288

Rees, M. J. 1984, ARA\&A, 22, 471

Shapiro, S. L., Lightman, A. P., \& Eardley, D. M. 1976, ApJ, 204, 187

Stern, B. E., Poutanen, J., Svensson, R., Sikora, M., \& Begelman, M. C. 1995, ApJ, 449, $\mathrm{L} 13+$ 
Sweet, P. A. 1958, in IAU Symposium, Vol. 6, Electromagnetic Phenomena in Cosmical Physics, ed. B. Lehnert, 123

Thorne, K. S. \& Price, R. H. 1975, ApJ, 195, L101

Uzdensky, D. \& Goodman, J. 2008, accepted to ApJ; ArXiv e-print:0803.0337

Uzdensky, D. A. 2007, ApJ, 671, 2139

van Oss, R. F., van den Oord, G. H. J., \& Kuperus, M. 1993, A\&A, 270, 275

Wood, J. H., Horne, K., Berriman, G., \& Wade, R. A. 1989, ApJ, 341, 974

Yamada, M., Ren, Y., Ji, H., Breslau, J., Gerhardt, S., Kulsrud, R., \& Kuritsyn, A. 2006, Physics of Plasmas, 13, 2119

Zdziarski, A. A. \& Gierliński, M. 2004, Progress of Theoretical Physics Supplement, 155, 99 